\title{
Classify arrhythmia by using 2D spectral images and deep neural network
}

\author{
Tran Anh Vu ${ }^{1}$, Hoang Quang Huy ${ }^{1}$, Pham Duy Khanh ${ }^{1}$, Nguyen Thi Minh Huyen ${ }^{1}$, \\ Trinh Thi Thu Uyen ${ }^{1}$, Pham Thi Viet Huong ${ }^{2}$ \\ ${ }^{1}$ Biomedical Engineering Department, School of Electronics and Telecommunications, Hanoi University of Science and Technology, \\ Hanoi, Vietnam \\ ${ }^{2}$ International School, Vietnam National University, Hanoi, Vietnam
}

\begin{abstract}
Article Info
Article history:

Received Jul 21, 2021

Revised Dec 8, 2021

Accepted Dec 18, 2021

\section{Keywords:}

2-D spectral image

Continuous wavelet transform

Deep neural network

ECG signal

ABSTRACT

Electrocardiogram (ECG) is the most common method for monitoring the working of the heart. ECG signal is the basis to determine normal or abnormal rhythm, thereby helping to accurately diagnose cardiovascular diseases. Therefore, an automatic algorithm to detect and diagnose abnormal heart rhythms is essential. There are many methods of classifying arrhythmias using machine learning algorithms such as k-nearest neighbors $(\mathrm{KNN})$, support vector machines (SVM), based on the features extracted from the record of ECG signal. Actually, deep learning algorithms are evolving and highly effective in image analysis and processing. In this research, a dense neural network model is proposed to classify normal and abnormal beats. Input ECG signal presenting a time series is converted into 2-D spectral image by applying wavelet transform. Our research is evaluated based on using the Massachusetts Institute of Technology-Beth Israel Hospital (MIT-BIH) arrhythmia database. The accuracy of the classification algorithm we employ is $99.8 \%$, demonstrating the model's validity when compared to other reports' findings. This is the foundation for our algorithm to prove it can be utilized as an efficient model for categorizing arrhythmia using ECG signals.
\end{abstract}

This is an open access article under the CC BY-SA license.

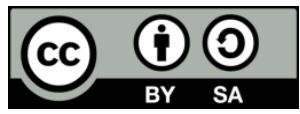

\section{Corresponding Author:}

Pham Thi Viet Huong

International School, Vietnam National University

144 Xuan Thuy, Cau Giay, Hanoi, Vietnam

Email: huongptv@isvnu.vn

\section{INTRODUCTION}

An arrhythmia [1] is an electrical irregularity of the heart, which can be a pacing or electrical conduction anomaly in the heart chambers, in which the heartbeat is irregular, too fast or too slow. An arrhythmia can be asymptomatic or cause symptoms such as palpitations, a sense that the heart is beating too quickly or irregularly, or a break between heartbeats [2]. Many cases of severe arrhythmias cause the patient to become dizzy, faint, have trouble breathing, and have chest pain. Complications can occur such as stroke, heart failure, or sudden death. According to WHO [3], cardiovascular diseases are the cause of the largest mortality in the world (more than 30\%), higher than death from cancer. It is estimated that each year about 17.9 million people worldwide die from cardiovascular diseases of which $85 \%$ are from heart attack and stroke. Especially in the current situation of COVID-19 epidemic, the risk of death often focuses mainly on the elderly or patients with underlying medical conditions including cardiovascular disease.

Electrocardiogram (ECG) is a chart that records the electrical impulses generated by cardiac muscle cell through electrodes placed in the body. The ECG signals are displayed in a 1-D time series that helps 
track and detect irregularities in the heart rhythm based on the waveform and the frequency of the heartbeat. Electrocardiograms can be used to diagnose cardiovascular problems in individuals. Electrocardiogram reading is a difficult task that needs experience and training. The specialist can evaluate if a clinical symptom of a heart problem is present based on the recorded data. As a result, identifying cardiac arrhythmias is mostly dependent on the knowledge of the doctor, and various doctors will provide different outcomes. Furthermore, with a lengthy time interval ECG record, young medical practitioners may overlook mild signals of cardiovascular illness. As a result, we require a tool to assist clinicians in the analysis of ECGs. As a result, we require a tool to assist clinicians in the analysis of ECGs. In which one of the key factors for properly diagnosing heart-related illnesses is the categorization of abnormal heart beats.

The ECG signal is a 1-D time series that can be processed and analyzed automatically by machine learning algorithms. Furthermore, deep learning algorithms have recently been demonstrated to be extremely efficient in the processing and categorization of 2D images. Deep learning algorithms, which are a subset of machine learning, rely on data to understand how to solve problems. Deep learning employs the neural network, a multi-layered structure of algorithms. Artificial neural networks offer unique characteristics that allow deep learning models to accomplish tasks that machine learning models have limitations.

There have been several studies in the subject of automated categorization arrhythmias. Wave morphological characteristics [4]-[6], as well as parameters such as variance and standard deviation [7] [8], have been extracted from 1-D ECG signals in the past, with the use of machine learning techniques such as KNN, support vector machine, decision tree, and random forest [9]-[11]. In order to extract the features or normalize data [7] most correctly, these techniques require a signal preprocessing step to filter noise, filter baseline drift [1]-[9]. Deep learning approaches are increasingly being used in image processing and analysis with great efficiency and accuracy [12]-[16]. The neural network model may operate effectively with multidimensional inputs without the feature extraction step. However, because the output of a 1-D input signal is less reliable than a 2-dimensional input, deep learning models often use a 2D picture as their input. A previous study [17] utilized a picture of the ECG signal that had not been transformed, which obtained 99.21\% accuracy rate. The input ECG (1-D) time series signal may be converted into a 2-D spectral picture using transformation techniques. Some recent research used a transformed 2D spectral image as the input of neural network classification model for 3 classes classification [15] with the accuracy of $98.7 \%$, and 8 classes classification [16] achieving an accuracy of $99.11 \%$.

Our contribution focuses primarily on approaches for extracting characteristics from an ECG signal and then doing classification using standard machine learning models, which yielded encouraging results. In addition to obtaining features in the time domain [4]-[6], some approaches employ transformation algorithms such as the Fourier transform and the wavelet transform to extract more characteristics of the signal in the frequency domain [7]-[9]. However, if linear features are present, performing feature extraction is extremely difficult and might reduce the classification model's effectiveness. Furthermore, if the database size is huge, standard machine learning methods will not attain the optimum efficiency. Heartbeat classification approaches based on deep learning algorithms have recently been presented as a solution to this challenge. The input processing of the neural network is also taken into consideration, in addition to the usage of multilayer neural network models with superior image classification efficiency. Only information about the waveforms is obtained when the classification model's input is an image of a 1D ECG signal [17], and this information is locally represented on the image, which means that, aside from the morphology of the signal, the remaining intervals on the image contain no information. As a result, several approaches have transformed a 1D ECG signal into a 2D spectral picture using transformation algorithms [15], [16]. The signal's temporal and frequency domain information are both contained in 2D spectral images. Clipping the signal segments at specific intervals from the beginning to the conclusion of the signal, on the other hand, might produce unevenness in the classification of the beats in the $2 \mathrm{D}$ pictures. We suggest a new approach in this study that is based on the evolution of earlier methods, which are:

a) Equally cut the signal segments by taking the same interval on both sides of the R peaks.

b) Using the continuous wavelet transform (CWT) to convert the signal segment information into $2 \mathrm{D}$ spectral pictures from the clipped signal segments.

c) The dense neural network model is utilized to identify heartbeats using these images as input.

d) Our research paper is organized. Part II explains our research method. Part III presents experimental results, and Part IV concludes the paper.

\section{RESEARCH METHOD}

\subsection{Databases}

In this study, we evaluate the effectiveness of our algorithm based on using the MIT-BIH arrhythmia database [1] which is published on Physionet.org. The database includes 48 ECG records, each is 
slightly more than 30 minutes long. Inside the database, each record of each different patient is bandwidth filtered at the frequency range of $0.1-100 \mathrm{~Hz}$ and digitized at a frequency of $360 \mathrm{~Hz}$. The records were labeled with the $\mathrm{R}$ peaks and the position of the peaks that appeared to be an arrhythmia. Therefore, the effectiveness of our classification model can be assessed. The three components of an ECG are depicted in Figure 1. The P wave represents atria depolarization; the QRS complex, which represents ventricular depolarization; and the $\mathrm{T}$ wave, which shows ventricle repolarization.

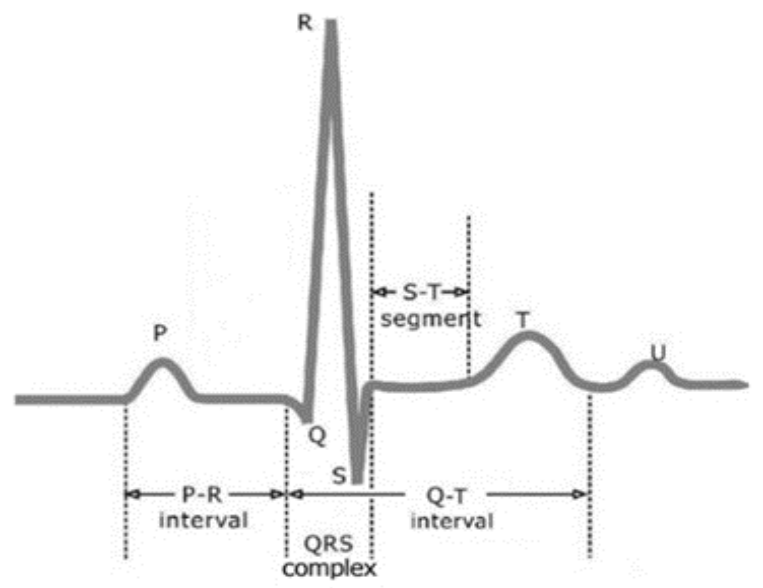

Figure 1. ECG of a heart in normal sinus rhythm

\subsection{Block diagram}

Figure 2 shows the implementation of the algorithm. The ECG signal is classified into two classes: normal and abnormal. Firstly, we use the package waveform-database (WFDB) for loading ECG and annotations from the dataset in Keras framework in Python. Each type is identified by a symbol that corresponds to the number of beats. The signal's peak R may be determined using the peak's characteristics. However, we utilize $\mathrm{R}$ peak value labeled in the dataset for signal processing simplicity. The second stage is signal segmentation, which involves taking an equal time-series signal before and after $\mathrm{R}$ peaks. Wavelet transformation is used to convert these segmented ECG signal intervals from time series to 2-dimensional spectral pictures. In this step, we use the scalogram tool to represent the transformed images. This image dataset is then used as the input of the classification deep neural network model. The image is divided into training sets and validation sets to perform classification and evaluate our model.

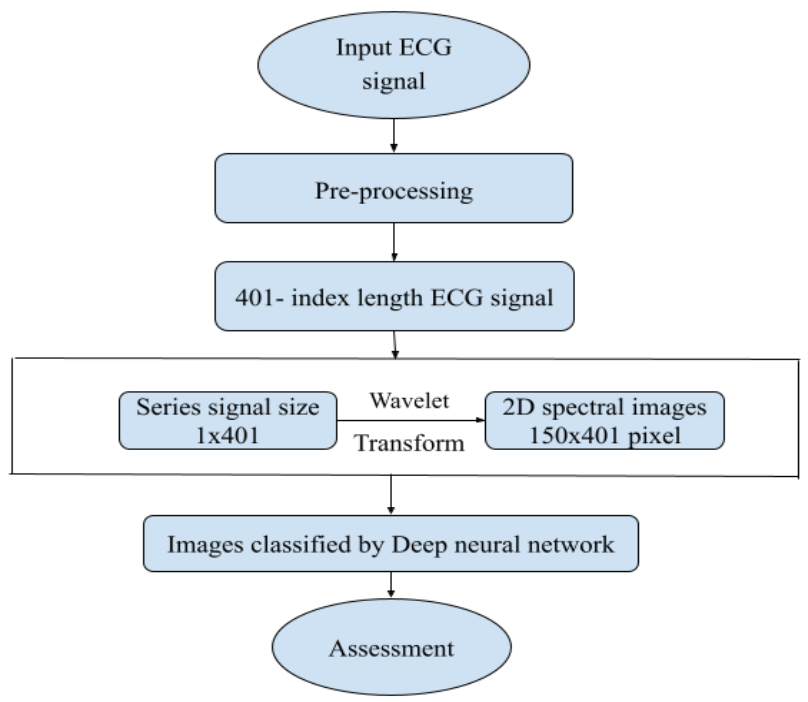

Figure 2. Arrhythmia detection diagram 


\subsection{Proposed method}

\subsubsection{Pre-processing}

To convert the signal ECG into images, we first need to output the R peak in the signal records, which is used to represent a heartbeat. We detect $\mathrm{R}$ peaks by signal's detecting peaks algorithm from the Scipy package based on properties of ECG signal's peak. The algorithm performs finding all local maxima in the data series by simply comparing neighboring values. Then select $\mathrm{R}$ peaks as a subset of these peaks based on the conditions of the peak's properties. The ECG signal in the data set has a sampling frequency of 360 $\mathrm{Hz}$, so the signal will be represented in the time domain by time index unit with each time index equal to $1 / 360$ s. In our case, we detect $R$ peaks by choosing the maximal value of the ECG signal in the minimum horizontal distance of 150 indexes between neighboring peaks. Figure 3 presents the R peaks of the ECG signal.

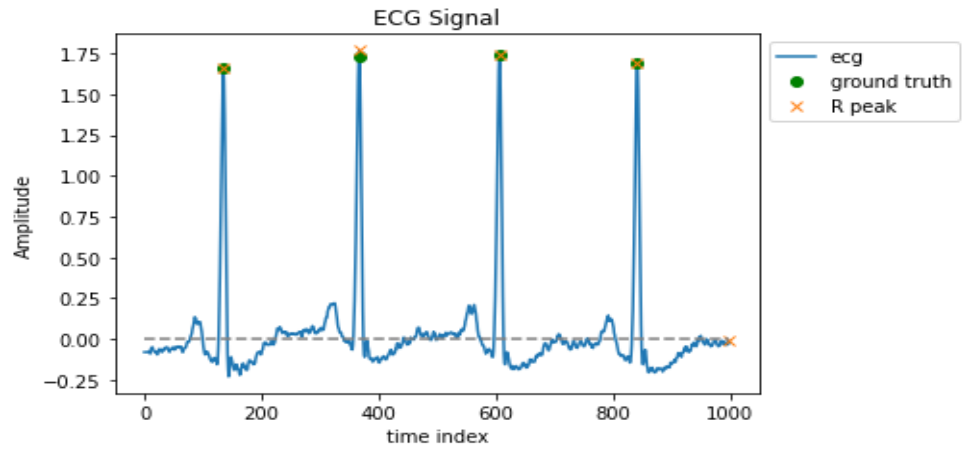

Figure 3 . R peaks detected by the scipy package

In this study, for simplicity, we utilize $\mathrm{R}$ peaks value, which has already been positioned in the database. From the data set, 9000 heartbeats are randomized with equal numbers of normal and irregular beats, for a total of 4500 beats. From the position of R peak of each beat, it will go backward and forward to each side a signal interval of the length equal 200 indexes.

\subsubsection{Generation of 2-D spectral images}

Theoretically, any signal can be decomposed into its component signals in both the temporal and the frequency domain. Therefore, the ECG signal can be analyzed into component signals to determine when and at what frequency the arrhythmia occurs [18]. The wavelet transform fulfills these two requirements. It makes the continuous signal $\mathrm{x}(\mathrm{t})$ from one dimension in two a 2D space defined as (1),

$$
\mathrm{S}(\mathrm{a}, \mathrm{b})=\frac{1}{\sqrt{a}} \int_{-\infty}^{+\infty} x(t) \Phi\left(\frac{t-b}{a}\right) d t
$$

where $\mathrm{a}$ and $\mathrm{b}$ are the scale factor and shift translation applied in the continuous parent wavelet $\Phi(\mathrm{t})$. In this step, the continuous wavelet transform (CWT) is applied to generate an ECG signal into a 2D spectrum. Depending on the study goal, several types of wavelet transforms can be employed to analyze ECG signals. For example, to remove ECG baseline, we use five wavelet transform families with a total of 14 wavelet configurations: Daubechies, Coiflets, Symlets, Fejer-Korovkin, and Meyer [19]. The variation of the abnormal heartbeat is a non-stationary signal so it is suitable to choose Morlet as the mother wavelet because of its analysis application on discriminate arrhythmias in the ECG signal [20]. defined as (2),

In theory, the Morlet wavelet is the most popular complex wavelet used in practice [21] and is

$$
\psi(t)=\frac{1}{\sqrt[4]{\Pi}}\left(e^{i \omega_{0} t}-e^{-\frac{\omega_{0}^{2}}{2}}\right) e^{\frac{-t^{2}}{2}}
$$

where $\omega_{0}$ is the central frequency of the mother wavelet and set by default for each wavelet with respective value. The second term in the bracket is correct for the non-zero mean of the complex sinusoid of the first term and can be negligible if $\omega_{0}>5$.

With regards to the scale factor, the size of the picture's height has an impact on the resolution of 2D spectral pictures. On the other side, the signal length corresponds to the width size of spectral images. We chose the features condition that offer the output pictures size with the best classification accuracy by running 
the experiment multiple times with varied sizes of $2 \mathrm{D}$ output images and comparing the classification results in part 3.

The scale factor is selected to transform linearity from 1 to 150 . The scale represents the number of times the wavelet is stretched. The larger scale, the more stretched wavelet is, and the more sensitive it is to lower signal frequencies. For better visualization, the scalogram is used to generate and show the 2D spectrum for the CWT. The CWT coefficients of a signal are taken in absolute value and its graph is plotted. Figure 4 presents an ECG signal and its scalogram.
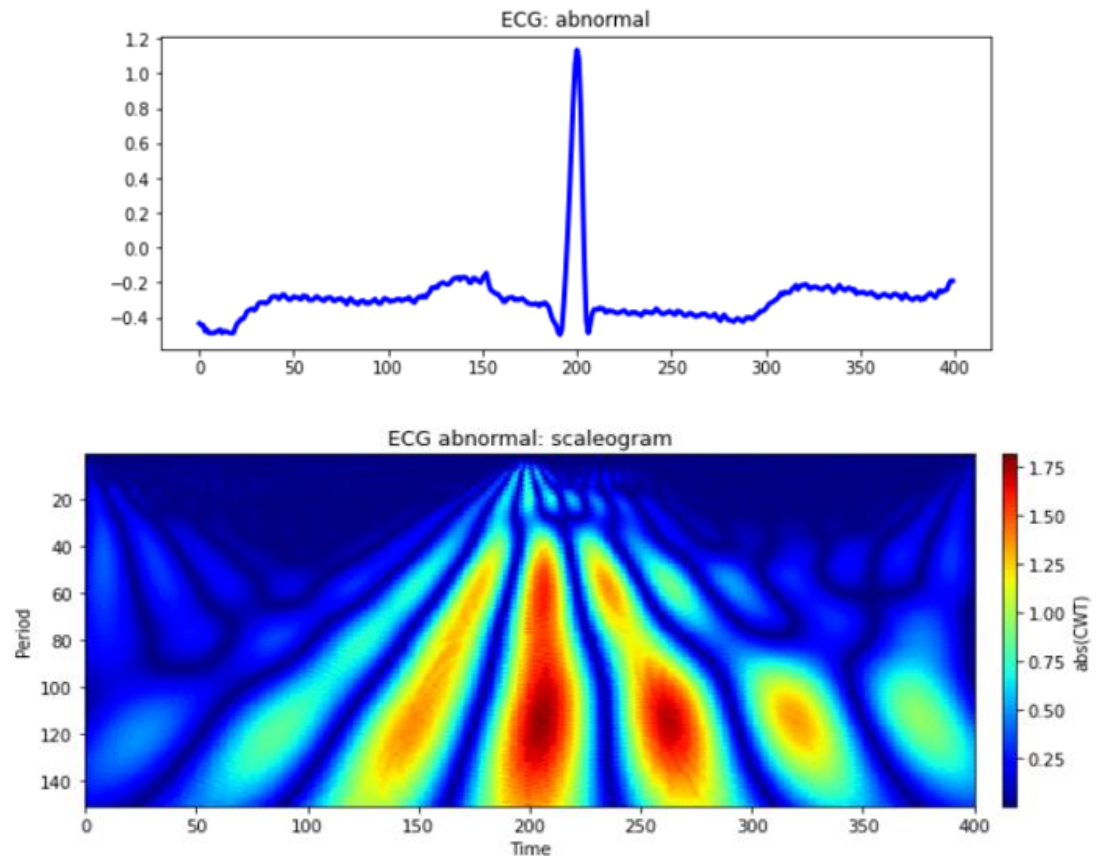

Figure 4. An ECG signal and its scalogram

In the scalogram output, the period in the vertical is defined by (3),

$$
\text { Period }=\frac{s}{b}
$$

where $\mathrm{s}$ is the scale, and $\mathrm{b}\left(\omega_{0}\right)$ is the central frequency used to build the chosen wavelet. Each horizontal feature may be regarded as a frequency of the total signal, and there is no continuous line in the output image to indicate that the frequencies are not time-consistent. The scalogram is a two-dimensional picture of 150x401 pixels, with 150 representing the number of scales used in the wavelet transform and 401 being the number of indices in the ECG signal data.

\subsubsection{Deep neural network construction}

Data setup: Our prepared dataset now is divided into the training set and test set with a 3/1 ratio; the train/test splits are generated to ensure that there is no overlap between the two sets.

Model construction: After the ECG signal is converted into a spectral image, each image will be assigned a value of 0 (normal peak) or 1 (abnormal peak). To classify these images, we use a basic convolutional neural network. The size of the model input is a x $150 \mathrm{x} 401$ where a is the number of $150 \mathrm{x} 401$ pixel images entered into the model. The hidden layer is constructed by two dense layers with 500 and 100 nodes separately. The output layer has two neurons for the final classifier that are either 0 (normal peak) or 1 (abnormal peak).

Activation function: The activation function may be used to compute the output of each node in an artificial neural network given a collection of image inputs. The rectified linear activation function (ReLU) is used in the hidden layer and is suggested as the default for multilayer perceptron (MLP) and convolutional neural networks (CNNs) [22]. If the input is positive, it will be directly output; otherwise, it will be zero. We use the Softmax activation function in the output layer to generate a vector of classification probabilities, 
with the probabilities of each value proportional to the relative scale of each value $(0$ and 1$)$ in the vector using (4),

$$
\sigma(z)_{i}=\frac{e^{z_{i}}}{\sum_{j=1}^{K} e^{z_{i}}}
$$

where $\mathrm{K}$ is the number of classes.

By applying the standard exponential function to each element $z_{i}$ and normalizes these values by dividing by the sum of all these exponentials, it ensures that each component will be in the interval $[0,1]$ and the sum of components output vector $z$ is 1 . After that, the class with higher probabilities value will be labeled as the image's type. Figure 5 presents the dense neural network (DNN) model architecture.

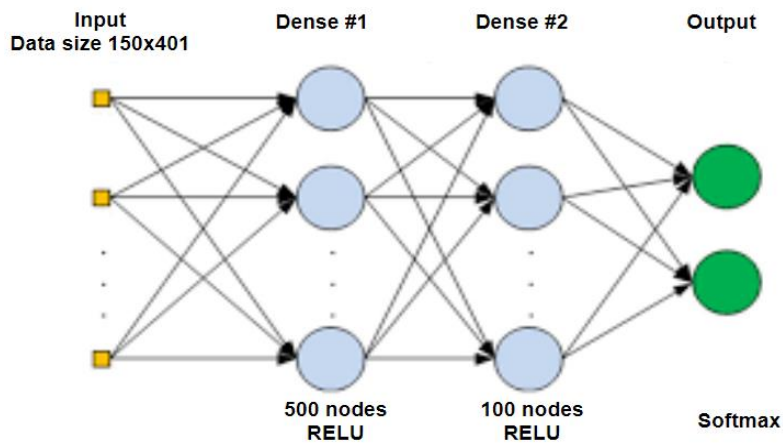

Figure 5. The dense neural network (DNN) model architecture

Cost function: The goal of the cost function is to compromise the accuracy of the algorithm, by taking the average error between the prediction result and the performance result. In theory, there are a variety of cost functions that can be used. In our paper, we choose sparse categorical cross-entropy as cost function because it saves memory and computation time. Instead of using an entire vector, it just utilizes a single integer. The cross-entropy loss between the labels and our results is calculated with the (5),

$$
\mathrm{C}=\frac{-1}{n} \sum_{c=1}^{N}\left(\left[\left(y_{c} * \ln \ln \left(a_{c}\right)+\left(1-y_{c}\right) \ln \ln \left(1-a_{c}\right)\right]\right)\right.
$$

where $\mathrm{C}$ is the cost to be minimized, $\mathrm{n}$ is the number of training points, $\mathrm{y}$ is the target value, $\mathrm{N}$ is the number of the classes, $\mathrm{c}$ is the index of the class, and a is the actual value. We use the stochastic gradient descent (SGD) optimizer for training our model. It evaluates the error gradient for the current state of the model using the training dataset, then updates the weights of our model via backpropagation.

\section{RESULTS AND DISCUSSION}

\subsection{Classification result}

The two parameters in our method that affect the result directly are scale of wavelet transform and the interval of signal for each spectral image. The large scale can offer the model high sensitivity, but it takes a long time to classify the data and the speed of the process is very slow. The signal segment intervals are similar. Long interval carries more information of ECG signal, but it also takes more time and decreases the speech of the process. With the capacity of our setup system, we have to tradeoff between the scales and the interval values.

Table 1 represents the parameters and the corresponding testing time. We have the best accuracy with a CWT scale of 150 and an ECG signal segment interval of 401 indices. The high sensitivity of the transformation model is shown by the scale value of 150. The signal interval from $\mathrm{R}$ peaks is 401 indexes before and after that there is adequate information in the present period and comparing the current period to the before and after periods.

The accuracy of the model is $99.8 \%$ after running 10 epochs using an optimizer of stochastic gradient descent and computing the loss with a sparse categorical cross-entropy. In the last step, we use the confusion matrix to evaluate our model. The confusion matrix allows us to evaluate the classification model visually. Each row represents the actual or true value, and each column represents the predicted value; we next compare the actual and predicted values for each class. The diagonal values are the biggest in an 
efficient model, equivalent to the number of predicted values equal to the actual value. The values in the matrix are then normalized to a range of 0 to 1 , with 1 being the desired value in the diagonal cells. Figure 6 presents the confusion matrix for the proposed classification model.

Table 1. Table of parameters and results in testing times

\begin{tabular}{cccc}
\hline Scale & Interval & Samples & Accuracy \\
\hline 50 & 401 & 9,000 & $99.16 \%$ \\
100 & 201 & 9,000 & $99.51 \%$ \\
100 & 201 & 9,000 & $99.73 \%$ \\
100 & 601 & 9,000 & $99.69 \%$ \\
150 & 201 & 9,000 & $99.20 \%$ \\
150 & 401 & 9,000 & $99.81 \%$ \\
\hline
\end{tabular}

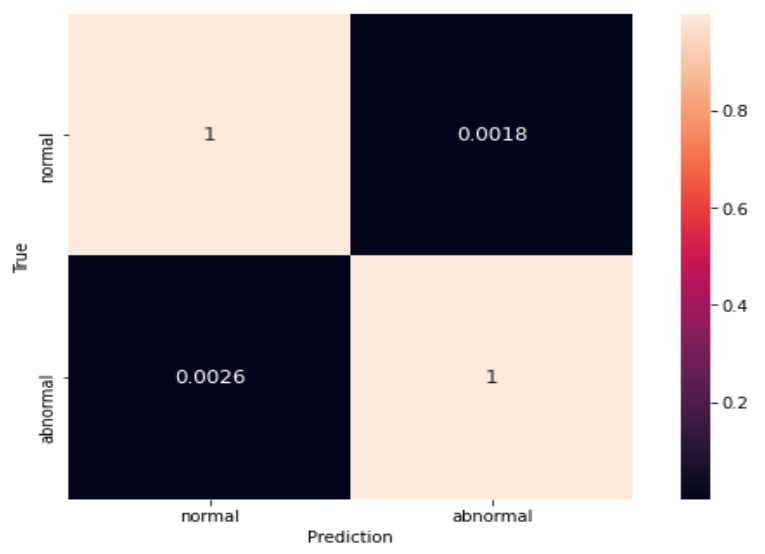

Figure 6. Confusion matrix for the proposed classification model

Our model has a confusion matrix with cells in diagonal equal 1, that show the amounts of actual abnormal beats and predicted abnormal beats almost similar. The learning curve in Figure 7 shows the effectiveness of this classification model. The learning curve shows the graphs of the value of loss function and accuracy of training set and validation set during the classification time. In our model, we receive good results in both training set and validation set. After the first epoch, the accuracy of the training set and validation set are very high and stable, approximately 1 . Contrary to the accuracy, the values of the loss function of two sets are very low and stable after the first epoch, approximately 0 . That proof our model is not overfitting or underfitting.

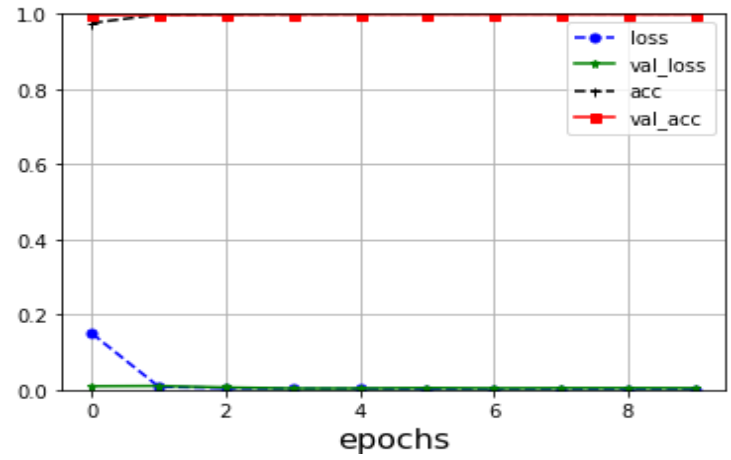

Figure 7. Learning curves

\subsection{Discussion}

To recognize the effectiveness of the method which uses wavelet transform to convert ECG signal to 2- D spectral images then classification by dense neural network model, we compare it with other methods also detecting arrhythmias and using MIT-BIH database. We compared the model of automatic arrhythmia classification based on ECG signaling with other recent models as shown in Table 2. Our average accuracy, 
sensitivity, specificity, precision value, in turn, reached 99.8\%, 99.7\%, 99.8\%, 99.8\% respectively, demonstrated superior performance when compared to previous algorithms that produced two classes in the five initial models. Our model has the highest average accuracy of the algorithms compared. This shows the superiority of deep learning algorithms compared to machine learning algorithms [9], [23], [24]. With other models using CNN or long short term memory (LSTM) [25], general sparsed neural network (GSNN) [26], radial basis function (RBF) [27], our model has better results, as our input to neural network model is 2D spectral images which have information in both time and frequency domain. One of the possible factors affecting the final result is the lesser number of heartbeats we utilize for training and testing our deep neural network model. When we develop our model to be a multiclass classification model, the results of seven models with output of more than two classes show that our model may be a good improvement.

The detection of arrhythmias on hourly long ECG records is time-consuming and requires the examiner to pay close attention. It is feasible to improve the performance of medical professionals by guiding the observer to analyze noticeable anomalies using automated categorization methods. As a result, the diagnosis and treatment of cardiovascular disorders in the clinic may be done faster and more efficiently.

Table 2. Comparison between the proposed model and other state-of-the-art ECG classification techniques

\begin{tabular}{lccccccc}
\hline Years & Model & Class & Accuracy $\%$ & Specificity $\%$ & Sensitivity $\%$ & Precision \% & F1 score \\
\hline 2018 & SVM [23] & 2 & $96 \%$ & - & - & - & - \\
2018 & KNN [9] & 2 & $97.5 \%$ & - & - & - & - \\
2019 & CNN [25] & 2 & $97.2 \%$ & $98.7 \%$ & $93.8 \%$ & $96.8 \%$ & - \\
2019 & LSTM [25] & 2 & $71.4 \%$ & $50.1 \%$ & $93.6 \%$ & $64.2 \%$ & - \\
2019 & SVM [24] & 2 & $98.3 \%$ & $97.5 \%$ & $99.1 \%$ & - & $98.3 \%$ \\
2021 & Proposed model (DNN) & 2 & $99.8 \%$ & $99.8 \%$ & $99.7 \%$ & $99.8 \%$ & - \\
2020 & GSNN [26] & 5 & $98 \%$ & - & - & $98 \%$ & - \\
2016 & SVM-RBF [27] & 5 & $98.91 \%$ & $97.85 \%$ & $98.91 \%$ & - & - \\
2019 & Faster R-CNN [17] & 5 & $99.21 \%$ & $99.45 \%$ & $98.06 \%$ & - \\
2020 & CNN [28] & 5 & $98.33 \%$ & $99.09 \%$ & $98.33 \%$ & $98.34 \%$ & - \\
2020 & LSTM [29] & 5 & $99.37 \%$ & $99.14 \%$ & $94.89 \%$ & $96.73 \%$ & $95.77 \%$ \\
2019 & CNN [30] & 5 & $99 \%$ & - & - & - & - \\
2020 & CNN [16] & 8 & $99.11 \%$ & $99.61 \%$ & $97.91 \%$ & $98.58 \%$ & $98 \%$ \\
2021 & CNN [15] & 3 & $98.7 \%$ & - & - & - & - \\
\hline
\end{tabular}

\section{CONCLUSION}

In this paper, we show how to use a dense neural network model to detect arrhythmias from ECG data recordings. An accurate taxonomy of ECG signals provides an excellent foundation for cardiovascular disease diagnosis and prognosis. Our approach is unique in that it uses the Wavelet transform to turn a onedimensional ECG signal into two-dimensional spectral pictures, which are then used as input to a classification model. When compared to methods that integrate feature extraction and current machine learning technologies, the neural network model has shown beneficial in enhancing the accuracy of heartbeat diagnoses.

\section{REFERENCES}

[1] C. Antzelevitch and A. Burashnikov, "Overview of basic mechanisms of Cardiac Arrhythmia," in Cardiac Electrophysiology Clinics, vol. 3, no. 1, pp. 23-45, 2011, doi: 10.1016/j.ccep.2010.10.012.

[2] G. B. Moody and R. G. Mark, "The impact of the MIT-BIH Arrhythmia Database," in IEEE Engineering in Medicine and Biology Magazine, vol. 20, no. 3, pp. 45-50, May-June 2001, doi: 10.1109/51.932724.

[3] WHO, "Cardiovascular diseases (CVDs)," who.int, https://www.who.int/en/news-room/fact-sheets/detail/ cardiovasculardiseases-(cvds) (accessed Dec. 2021)

[4] M. Hammad, A. Maher, K. Wang, F. Jiang, and M. Amrani, "Detection of abnormal heart conditions based on characteristics of ECG signals," Measurement, vol. 125, pp. 634-644, 2018, doi: 10.1016/j.measurement.2018.05.033.

[5] M. K. Senapati, M. Senapati, and S. Maka, "Cardiac arrhythmia classification of ECG signal using morphology and heart beat rate," 2014 Fourth International Conference on Advances in Computing and Communications, 2014, pp. 60-63, doi: 10.1109/ICACC.2014.20.

[6] P. Chazal, M. O'Dwyer, and R. B. Reilly, "Automatic classification of heartbeats using ECG morphology and heartbeat interval features," in IEEE Transactions on Biomedical Engineering, vol. 51, no. 7, pp. 1196-1206, July 2004, doi: 10.1109/TBME.2004.827359.

[7] H. M. Rai, A. Trivedi, and S. Shukla, "ECG signal processing for abnormalities detection using multi-resolution wavelet transform and artificial neural network classifier," Measurement, vol. 46, no. 9, pp. 3238-3246, 2013, doi: 10.1016/j.measurement.2013.05.021

[8] A. Diker, D. Avci, E. Avci, and M. Gedikpinar, "A new technique for ECG signal classification genetic algorithm Wavelet Kernel extreme learning machine," Optik, vol. 180, pp. 46-55, 2019, doi: 10.1016/j.ijleo.2018.11.065.

[9] C. Venkatesan, P. Karthigaikumar, and R. Varatharajan, "A novel LMS algorithm for ECG signal preprocessing and KNN classifier-based abnormality detection," Multimedia Tools and Applications, vol. 77, pp. 10365-10374, 2018, doi: $10.1007 / \mathrm{s} 11042-018-5762-6$. 
[10] C. Venkatesan, P. Karthigaikumar, A. Paul, S. Satheeskumaran, and R. Kumar, "ECG Signal Preprocessing and SVM ClassifierBased Abnormality Detection in Remote Healthcare Applications," in IEEE Access, vol. 6, pp. 9767-9773, 2018, doi: 10.1109/ACCESS.2018.2794346.

[11] P. Shimpi, S. Shah, M. Shroff, and A. Godbole, "A machine learning approach for the classification of cardiac arrhythmia," 2017 International Conference on Computing Methodologies and Communication (ICCMC), 2017, pp. 603-607, doi: 10.1109/ICCMC.2017.8282537.

[12] E. Izci, M. A. Ozdemir, M. Degirmenci, and A. Akan, "Cardiac Arrhythmia Detection from 2D ECG Images by Using Deep Learning Technique," 2019 Medical Technologies Congress (TIPTEKNO), 2019, pp. 1-4, doi: 10.1109/TIPTEKNO.2019.8895011.

[13] G. Swapna, P. Somank, and R. Vinayakumar, "Automated Detection of Cardiac Arrhythmia using Deep learning Techniques," Procedia Computer Science, vol. 132, pp. 1192-1201, 2018, doi: 10.1016/j.procs.2018.05.034.

[14] R. Rohmantri and N. Surantha, "Arrhythmia Classification using 2D Convolutional Neural Network," International Journal of Advanced Computer Science and Applications (IJACSA), vol. 11, no. 4, pp. 201-208, 2020, doi: 10.14569/IJACSA.2020.0110427.

[15] R. F. Olanrewaju, S. N. Ibrahim, A. L. Asnawi, and H. Altaf, "Classification of ECG signals for detection of arrhythmia and congestive heart failure based on continuous wavelet transform and deep neural networks," Indonesian Journal of Electrical Engineering and Computer Science (IJEECS), vol. 22, no. 3, June 2021, pp. 1520-1528, doi: 10.11591/ijeecs.v22.i3.pp1520-1528.

[16] A. Ullah, S. Anwar, M. Bilal, and R. M. Mehmood, "Classification of Arrhythmia by using Deep Learning with 2D ECG spectral image representation," Remote Sensing, vol. 12, 1685, 2020, doi: 10.3390/rs12101685.

[17] Y. Ji, S. Zhang, and W. Xiao, "Electrocardiogram Classification Based on Faster Regions with Convolutional Neural Network," Sensors (Basel), vol. 19, no. 11, p. 2558, 2019, doi: 10.3390/s19112558.

[18] C. Herff and D. J. Krusienski, "Extracting Features from Time Series," In: Kubben P., Dumontier M., Dekker A. (eds) Fundamentals of Clinical Data Science. Springer, Cham., pp. 85-100, 2019, doi: 10.1007/978-3-319-99713-1_7.

[19] C. Chen and F. R. Tsui, "Comparing different wavelet transforms on removing electrocardiogram baseline wanders and special trends," BMC Medical Informatics and Decision Making, vol. 20, no. S11, p. 343, 2020, doi: 10.1186/s12911-020-01349-x.

[20] O. P. Neto et al., "Morlet wavelet transforms of heart rate variability for autonomic nervous system activity," Applied and Computational Harmonic Analysis, vol 40, no. 1, pp. 200-206, 2016, doi: 10.1016/j.acha.2015.07.002.

[21] P. S. Addison, "Wavelet transforms and the ECG: A review," Physiological measurement, vol. 26, no. 5, pp. R155-R199, 2005, doi: 10.1088/0967-3334/26/5/R01.

[22] J. Brownlee, "A Gentle Introduction to the Rectified Linear Unit (ReLU)," January 9, 2019, Deep Learning Performance. https://machinelearningmastery.com/rectified-linear-activation-function-for-deep-learning-neural-networks (accessed Dec. 15, 2021).

[23] C. Venkatesan, P. Karthigaikumar, A. Paul, S. Satheeskumaran, and R. Kumar, "ECG Signal Preprocessing and SVM ClassifierBased Abnormality Detection in Remote Healthcare Applications," in IEEE Access, vol. 6, pp. 9767-9773, 2018, doi: 10.1109/ACCESS.2018.2794346.

[24] M. K. Moridani, M. A. Zadeh, and Z. S. Mazraeh, “An Efficient Automated Algorithm for Distinguishing Normal and Abnormal ECG Signal," IRBM, vol. 40, no. 6, pp. 332-340, 2019, doi: 10.1016/j.irbm.2019.09.002.

[25] A. Long, "Detecting Heart Arrhythmias with Deep Learning in Keras with Dense, CNN, and LSTM," https://towardsdatascience.com/detecting-heart-arrhythmias-with-deep-learning-in-keras-with-dense-cnn-and-lstm-add337d9e41f (accessed Nov 26, 2021).

[26] S. T. Sanamdikar, S. T. Hamde, and V. G. Asutkar, "Analysis and classification of cardiac arrhythmia based on general sparsed neural network of ECG signals," SN Applied Sciences 2, p. 1244, 2020, doi: 10.1007/s42452-020-3058-8.

[27] F. A. Elhaj, N. Salim, A. R. Harris, T. T. Swee, and T. Ahmed, "Arrhythmia recognition and classification using combined linear and nonlinear features of ECG signals," Computer Methods and Programs in Biomedicine, vol. 127, pp. 52-63, 2016, doi: 10.1016/j.cmpb.2015.12.024.

[28] A. A. Bonab, M. C. Amirani, and A. Mehri, "Spectral entropy and deep convolutional neural network for ECG beat classification," Biocybernetics and Biomedical Engineering, vol. 40, no. 2, pp. 691-700, 2020, doi: 10.1016/j.bbe.2020.02.004.

[29] S. Pandey and R. R. Janghel, "Automatic arrhythmia recognition from electrocardiogram signals using different feature methods with long short-term memory network model," Signal, Image and Video Processing, vol. 14, no. 4, pp. 1255-1263, 2020, doi: $10.1007 / \mathrm{s} 11760-020-01666-8$.

[30] J. Huang, B. Chen, B. Yao, and W. He, "ECG Arrhythmia Classification Using STFT-Based Spectrogram and Convolutional Neural Network," IEEE Access, vol. 7, pp. 92871-92880, 2019, doi: 10.1109/ACCESS.2019.2928017.

\section{BIOGRAPHIES OF AUTHORS}

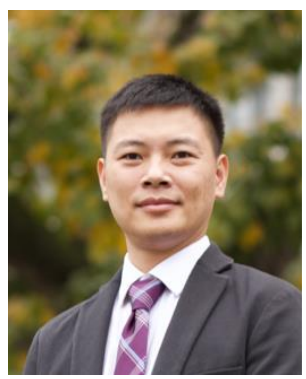

Dr. Tran-Anh Vu (D) S SC P is a senior lecturer and Vice Head of Biomedical Engineering Department, School of Electronics and Telecommunications, Hanoi University of Science and Technology, Hanoi, Vietnam. He earned his Ph.D. degree in Electrical Engineering from the University of Massachusetts at Lowell (USA) in 2014, MS degree in Biomedical Engineering from Tufts University (USA) in 2010, MS degree in Electronics and Telecommunication from Hanoi University of Science and Technology (Vietnam) in 2002, and BS degree in Electronics and Telecommunications from Hanoi University of Science and Technology (Vietnam) in 2000. His main research interests include the medical data analysis and classification, research and development applications for rehabilitation, smart health. He can be contacted at email: Vu.trananh@hust.edu.vn. 


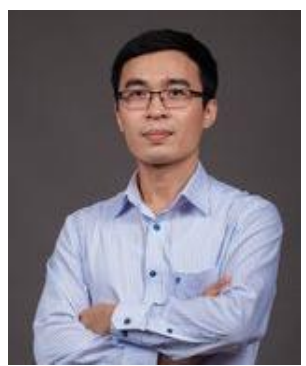

Hoang-Quang Huy (iD S SC P is a lecturer of Biomedical Engineering Department, School of Electronics and Telecommunications, Hanoi University of Science and Technology, Hanoi, Vietnam. He earned his MS degree in Electronics and Telecommunication from Hanoi University of Science and Technology (Vietnam) in 2006, and BS degree in Electronics and Telecommunications from Hanoi University of Science and Technology (Vietnam) in 2002. His main research interests include the medical data analysis and classification, research and development applications for rehabilitation, smart health, hospital information systems. He can be contacted at email: Huy.hoangquang@mail.hust.edu.vn.

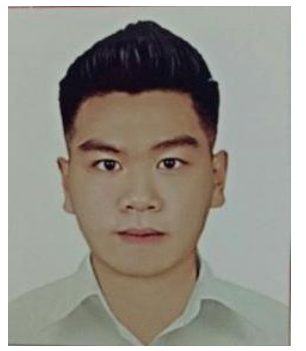

Pham Duy Khanh (D) SC SC P obtained his B.Sc in Biomedical Engineering Department, School of Electronics and Telecommunications, Hanoi University of Science and Technology, Hanoi, Vietnam in 2021. His main research interests include the medical data analysis and classification, research and development applications in Biomedical Engineering. He can be contacted at email: duykhanh030799bka@gmail.com.

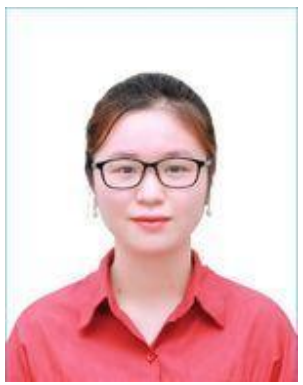

Nguyen Thi Minh Huyen (D) SC P obtained her B.Sc, in Biomedical Engineering Department, School of Electronics and Telecommunications, Hanoi University of Science and Technology, Hanoi, Vietnam in 2021, and now is a master student. Her main research interests include the medical data analysis and classification, research and development applications in Biomedical Engineering. She can be contacted at email: minhhuyennguyen121199@gmail.com.

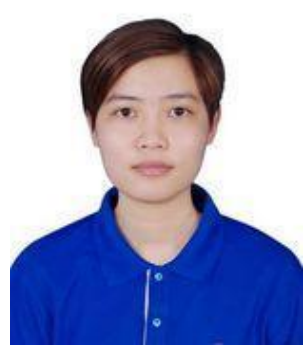

Trinh Thi Thu Uyen (D) SC P obtained her B.Sc, in Biomedical Engineering Department, School of Electronics and Telecommunications, Hanoi University of Science and Technology, Hanoi, Vietnam in 2021, and now is a master student. Her main research interests include the medical data analysis and classification, research and development applications in Biomedical Engineering. She can be contacted at email: trinhthuuyen326@gmail.com.

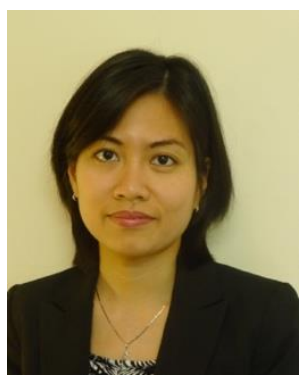

Dr. Pham-Thi-Viet Huong (iD 8 SC P obtained her B.Sc in Electrical Engineering from Hanoi University of Science and Technology in 2007. She got her MSc and PhD, both in Electrical Engineering, from University of Massachusetts Lowell in the United States, in 2010 and 2012. From 2012 to 2015, she was a researcher in the Manning School of Business, Lowell, Massachusetts. From 2017-2020, she was the faculty of VNU University of Engineering and Technology, Vietnam (VNU-UET). Since 2020, she works in International School-VNU. She is interested in data mining and analytics, machine learning methodologies, with applications in Biomedical Engineering. She can be contacted at email: Huongptv@isvnu.vn. 